\title{
Humanistic and economic burden of nausea and vomiting among migraine sufferers
}

\section{Kavita Gajria' \\ Lulu K Lee ${ }^{2}$ \\ Natalia M Flores ${ }^{2}$ \\ Ernesto Aycardi' \\ Sanjay K Gandhi'}

'Global Health Economics Research, Teva Pharmaceuticals, New York, NY, USA; ${ }^{2}$ Health Outcomes Practice, Kantar Health, Foster City, CA, USA
Correspondence: Lulu K Lee Health Outcomes Practice, Kantar Health, I8I0 Gateway Drive, Suite I20, San Mateo, CA 94404, USA

$\mathrm{Tel}+\mathrm{I} 6507202246$

Fax + I 2126477659

Email Lulu.Lee@Kantarhealth.com
This article was published in the following Dove Press journal: Journal of Pain Research

24 March 2017

Number of times this article has been viewed

Background: While studies have demonstrated the economic burden of migraines in terms of quality of life, health care resource use (HRU), and costs, there exists a notable paucity of data comparing such outcomes among migraineurs with nausea and vomiting $(\mathrm{N} / \mathrm{V})$ and those without. The current study aimed to address this gap.

Methods: This was a retrospective study using data from the 2013 US National Health and Wellness Survey, a cross-sectional, internet-based survey. Respondents self-reported their migraine with or without N/V along with demographics and outcomes including depression (Patient Health Questionnaire total score; PHQ-9), sleep problems (11-item total score of sleep problems), HRU (number of physician visits, emergency room [ER] visits, and hospitalizations) and Work Productivity and Activity Impairment-General Health Scale (WPAI-GH), and associated mean annual costs. Generalized linear models, adjusting for covariates, assessed the burden of N/V on all outcomes. Results: Among all migraineurs $(\mathrm{N}=7,855), 73.4 \%$ were female, mean age was 41.82 years old, and $57.6 \%$ reported experiencing N/V. Adjusting for covariates, migraineurs with N/V vs without N/V had higher mean PHQ-9 scores $(7.91$ vs $7.02, p<0.001)$ and mean sleep problems (3.29 vs $2.64, p<0.001)$. Mean ER visits were more frequent among migraineurs with $\mathrm{N} / \mathrm{V}$ than those without $\mathrm{N} / \mathrm{V}(0.48$ vs $0.38, p=0.001)$. This difference translated into a $26.3 \%$ increase in estimated mean ER costs (N/V=US $\$ 1,499$ vs without N/V=US $\$ 1,187, p=0.002$ ). Mean percentage activity impairment was higher in migraineurs with $\mathrm{N} / \mathrm{V}$ than in those without N/V $(37.73 \%$ vs $35.12 \%, p=0.002)$ and migraineurs with $\mathrm{N} / \mathrm{V}$ had higher work productivity loss costs $(\mathrm{N} /$ $\mathrm{V}=\mathrm{US} \$ 10,344$ vs without N/V=US $\$ 9,218, p=0.016$ ).

Conclusion: Migraine patients with N/V reported worse depression, sleep problems, and activity impairment, and higher ER visits than those without N/V. Migraine with N/V was also associated with an increase in mean annual ER visit costs and work productivity loss costs. Study findings suggest unmet needs with current treatment options for migraine patients with $\mathrm{N} / \mathrm{V}$.

Keywords: migraine, nausea, vomiting, depression, work productivity, healthcare resource use, costs

\section{Introduction}

Migraine is a neurological disorder that involves excitation and sensitivity of certain areas of the brain and is thought to possess a genetic underpinning. ${ }^{1,2}$ It is characterized by recurrent episodes of headache, often accompanied by related symptoms such as nausea, vomiting, photophobia, and phonophobia. Migraine attacks typically last for 4-72 hours and can be disabling, forcing people to miss work or other activities. ${ }^{3}$ Noteworthy in the context of the current study is that nausea and vomiting are experienced by the majority of patients, with estimates of nausea occurring in $90 \%$ of cases and vomiting in $70 \%{ }^{4}$ 
Studies suggest that prevalence rates of migraines in developed countries range from $18 \%$ to $18.2 \%$ among females and $6 \%$ to $6.5 \%$ among males. ${ }^{5-7}$ Importantly, migraine patients are at greater risk of experiencing a number of other comorbid conditions than the general population, including, for example, stroke, coronary heart disease, hypertension, epilepsy, and asthma. ${ }^{89}$ Extensive research has also established a consistent association between migraine and increased risk of psychiatric illness, such as symptoms of depression and anxiety. ${ }^{10-12}$ Additionally, it has been found that migraine patients have poorer sleep quality independent of depression or anxiety. ${ }^{13}$

In addition to managing comorbid medical and psychiatric conditions, the symptoms associated with migraine headache can be severe and can often require bed rest and medication. Migraine has thus been associated with substantial and ongoing impairments in quality of life and health outcomes among sufferers. ${ }^{14}$ A number of large-scale survey studies have established significant and persistent impairments in quality of life among migraine sufferers, including physical, social, functional, and emotional domains. ${ }^{15,16}$ However, research has also suggested that treatment and stable symptomatology can be associated with improved quality of life. ${ }^{17,18}$

Migraine-related impairments also represent a tangible economic burden, as migraineurs often miss work or school, have decreased productivity while at work, and often utilize health care resources at a greater frequency than nonmigraineurs. ${ }^{6}$ Indeed, headaches or head pain was the fourth leading cause of visits to the emergency room (ER) in the US in 2009-2010 and accounted for $0.5 \%$ of visits across all ambulatory care settings. ${ }^{19}$ Additionally, those with chronic migraine incurred significantly higher health care costs compared with those with episodic migraine. ${ }^{20}$

The effect on health and economic outcomes among migraineurs can also vary depending on the frequency and severity of symptoms. Those suffering from nausea and vomiting represent a subsample of patients who experience greater impairment in quality of life and incur a larger economic burden than those without these specific symptoms..$^{20,21}$ Further, a recent analyses from the AMPP study found that persistent nausea was associated with increased risk of progression to chronic migraine, reinforcing the importance of addressing this frequent symptomatology. ${ }^{22}$

The importance of addressing nausea and vomiting in the context of migraine is key for several reasons, one of which is that symptoms such as nausea and vomiting delay or interfere with treatments that are usually administered orally, prompting patients to visit ERs and clinics to seek alternative treatments. ${ }^{4}$ Despite this, a literature search identified only one survey study that investigated resource use and costs among subjects with or without nausea, thus suggesting a critical need for further research.

In a 2013 study examining the economic consequences of nausea among nearly 6,500 migraine sufferers in the US, Lipton et $\mathrm{al}^{20}$ reported a significant association between symptoms of nausea and increased economic burden. The authors found that approximately $50 \%$ of those surveyed reported headache-related nausea more than half the time. Outpatient care (ie, health care provider visits) costs for these individuals were more than triple than that for migraine sufferers without nausea. Migraine sufferers reporting nausea were significantly more likely to have utilized a range of health care resources in the past year, including primary care and the emergency department, further emphasizing the substantial economic burden associated with nausea among this patient group.

Whereas several studies have demonstrated the economic burden of migraines in terms of quality of life, health care resource use and costs, there exists a notable paucity of data comparing such outcomes among migraineurs with nausea and vomiting and those without. The current study seeks to address this gap by estimating the incremental burden associated with nausea/vomiting (N/V) on depression, sleep problems, health care resource use (HRU), work productivity and activity impairment, and related costs among migraineurs.

\section{Materials and methods \\ Data source}

This is a retrospective analysis of data from the 2013 US National Health and Wellness Survey (NHWS; N=75,000). The US NHWS is a self-administered, internet-based general health questionnaire surveying a sample of adults (aged 18 or older) that is fielded annually. The NHWS assesses health status, attitudes, behaviors, treatments used, and health outcomes (including patient-reported validated instruments) on a number of health conditions.

Respondents of the US NHWS are recruited from an internet panel using a stratified sampling framework to ensure the demographic composition (ie, age, gender, and ethnicity) is representative of the US adult population based on the United States Census Bureau. Among 75,000 total respondents in the NHWS, the study sample included those who self-reported a physician diagnosis of migraines $(\mathrm{N}=7,855)$. All respondents in the NHWS provided online written consent to participate. As the NHWS study is anonymized and the data 
are deidentified, this study was granted exemption status by the Essex Institutional Review Broad (Lebanon, NJ, USA).

All respondents with a self-reported physician diagnosis of migraines were asked to report specific symptoms they experienced as a result of their migraines from a symptoms checklist (eg, nausea, vomiting, etc). Respondents who selfreported having migraines with $\mathrm{N} / \mathrm{V}(\mathrm{n}=4,528)$ were compared with respondents who self-reported having migraines without N/V (n=3,327).

\section{Measures}

The following measures as detailed below were obtained from the NHWS.

\section{Demographics, health, and clinical characteristics} Demographics included age, gender, race/ethnicity, education, and household income. Health characteristics included body mass index (BMI) category, smoking status, alcohol use, and exercise behavior. The Charlson comorbidity index (CCI) was also examined for general comorbidity burden. ${ }^{23,24}$ The CCI weights the presence of the following conditions and provides a summed score: human immunodeficiency virus/acquired immune deficiency syndrome (HIV/AIDS), metastatic tumor, lymphoma, leukemia, any tumor, moderate/ severe renal disease, hemiplegia, diabetes, mild liver disease, ulcer disease, connective tissue disease, chronic pulmonary disease, dementia, cerebrovascular disease, peripheral vascular disease, myocardial infarction, congestive heart failure, and diabetes with end organ damage. Clinical characteristics included symptoms due to migraines (eg, aura, moderate to severe pain, sensitivity to light), severity of migraines (mild, moderate, severe), and current prescription treatment of migraines.

\section{Depression}

A summary depression severity score was computed for each respondent using the 9-item Patient Health Questionnaire (PHQ-9). ${ }^{25,26}$ The PHQ-9 is the 9-item depression module from the full PHQ and focuses on the nine diagnostic criteria for DSM-IV depression disorders. Scores on the PHQ-9 range from 0 to 27, with higher scores representing worse depression. There also exist thresholds with cut points of 5, 10, 15, and 20 representing mild, moderate, moderately severe, and severe depression, respectively. ${ }^{26}$

\section{Sleep difficulties}

Total sleep scores were computed by a summation of respondents' self-report of sleep difficulty experienced as yes/no response to 11-items. Respondents were asked to think about the sleeplessness or difficulty sleeping they experienced and select all sleep problems or symptoms they regularly experience. These items included: difficulty falling asleep, difficulty staying awake, daytime sleepiness, leg cramps/leg problems, night sweats/hot flashes, poor quality of sleep, sleep apnea, waking up to go to the bathroom, waking up too early (such as before the alarm clock), waking during the night and not being able to get back to sleep, and waking up several times during the night. Higher scores indicated greater sleep problems.

\section{Health care resource use (HRU)}

Frequencies of various forms of all-cause HRU in the past 6 months were reported, including emergency room (ER) visits, hospitalizations, and traditional health care provider (HCP) visits (includes general/family practitioner, internist, and specialists).

\section{Work productivity loss and activity impairment}

Work productivity loss and activity impairment were measured via the Work Productivity and Activity ImpairmentGeneral Health Scale (WPAI-GH). ${ }^{27}$ The WPAI-GH is a 6-item validated instrument consisting of four metrics: absenteeism (the percentage of work time missed because of one's health in the past 7 days), presenteeism (the percentage of impairment experienced while at work in the past 7 days because of one's health), overall work productivity loss (an overall impairment estimate that is a combination of absenteeism and presenteeism), and activity impairment (the percentage of impairment in daily activities because of one's health in the past 7 days). Higher scores represent greater work productivity loss and activity impairment. Only respondents who reported being employed full-time or part-time provided data for absenteeism, presenteeism, and overall work impairment. All respondents provided data for activity impairment.

\section{Direct and indirect costs}

Annual estimated direct costs included all-cause HRU costs and were extrapolated from the Medical Expenditure Panel Survey (MEPS) ${ }^{28}$ by applying 2012 age-specific unit cost to each HRU event of NHWS respondents. Similar extrapolation was conducted for annual indirect costs where 2012 age- and gender-specific wages from the Bureau of Labor Statistics $(B L S)^{29}$ were applied to work hours lost with presenteeism and absenteeism from the WPAI-GH. Estimated total costs were calculated as a sum of direct and indirect costs. For 
respondents who were unemployed, their estimated total costs did not include indirect costs.

\section{Statistical analyses}

Comparisons between migraineurs with $\mathrm{N} / \mathrm{V}$ and without $\mathrm{N} / \mathrm{V}$ were made on demographics and health characteristics using one-way ANOVAs (for continuous variables) and $\chi^{2}$ tests (for categorical variables). These analyses helped inform covariate selection for multivariable analyses. Variables with significant statistical differences $(p<0.05)$ between the two groups were adjusted for in the multivariable analyses. Unadjusted comparisons between migraineurs with N/V and without $\mathrm{N} / \mathrm{V}$ on health outcomes were also examined using one-way ANOVAs and $\chi^{2}$ tests.

Generalized linear models (GLMs) were then used to predict outcomes as a function of absence vs presence of $\mathrm{N} / \mathrm{V}$, controlling for covariates. Covariates included in all multivariable models were: age, gender (male vs female [reference group]), ethnicity (non-Hispanic black, Hispanic, other vs non-Hispanic white [reference group]), education (<4-year degree vs 4-year or more degree [reference group]), income (<US\$25,000, US\$25,000-US\$50,000, US\$50,000US\$75,000, declined to answer vs US\$75,000 or more [reference group]), BMI (underweight, overweight, obese, unknown vs normal weight [reference group]), CCI, exercise ( 0 times vs $1+$ times for 20 minutes per month [reference group]), smoker (current, former vs never [reference group]), and alcohol use (drinker vs nondrinker [reference group]).

GLMs specifying normal distributions were used to predict PHQ-9 summary scores and total sleep difficulty scores. GLMs specifying negative binomial distributions with loglink functions were used to predict the number of HRU visits, presenteeism, absenteeism, overall work impairment, activity impairment, and costs due to pronounced skew. Adjusted means were reported along with $95 \%$ confidence intervals (CIs). Any two-sided $p$-value less than 0.05 was considered statistically significant.

\section{Results}

\section{Sample demographics and characteristics}

Among the $\mathrm{N}=7,855$ migraineurs, $73 \%$ were female, $72 \%$ were white, mean age was 41.8 years $(\mathrm{SD}=14.0)$, mean CCI was $0.55(\mathrm{SD}=1.26)$, and mean number of migraine episodes reported in the past 30 days was $3.27(\mathrm{SD}=5.33)$ (Table 1$)$. Overall, those with N/V compared with those without N/V were slightly younger (41.2 vs 42.7 years old) and a higher proportion were female $(82.5 \%$ vs $61.0 \%)$, white $(75.3 \%$ vs $66.9 \%$ ), obese (BMI; 35.9\% vs 33.3\%), and had higher income ( $26.1 \%$ vs $23.0 \%$ for US $\$ 75,000$ or more), all $p<0.05$ (Table 1).

Among all migraineurs, 57.6\% reported experiencing N/V. Other common symptoms reported included moderate to severe pain (78.5\%), pulsating, throbbing, or pounding pain $(69 \%)$, and sensitivity to light (71.5\%) (Table 2$)$. The majority of migraineurs classified their migraine severity as moderate $(50.6 \%)$ or severe $(33.8 \%)$ (Table 2$)$. In terms of treatment, the three most common prescription medications used among migraineurs included sumatriptan tablets, topiramate, and rizatriptan (Table 2).

\section{Health and economic outcomes as a function of migraines with $N / V$ vs without N/V \\ Depression and sleep difficulties}

Unadjusted mean comparisons showed that migraineurs with N/V scored higher on PHQ-9 than those without N/V. Additionally, number of total sleep difficulties was higher among migraineurs with N/V compared with those without N/V.

Consistent with unadjusted comparison results, adjusted comparisons showed that migraineurs with $\mathrm{N} / \mathrm{V}$ vs those without N/V scored higher PHQ-9 depression scores (adjusted mean=7.91 [CI: 7.55-8.28] vs 7.02 [CI: 6.66-7.37], $p<0.001$ ) (Figure 1). Similarly, adjusted analyses showed that migraineurs with $\mathrm{N} / \mathrm{V}$ experienced greater number of total sleep difficulties than those without N/V (adjusted mean=3.29 [CI: 3.14-3.44] vs 2.64 [CI: 0.075-2.49], $p<0.001$ ) (Figure 2).

\section{Work productivity and activity impairment}

Unadjusted comparisons revealed no significant differences on absenteeism, presenteeism, and overall work productivity impairment between the migraine groups. However, migraineurs with $\mathrm{N} / \mathrm{V}$ experienced greater activity impairment than those without N/V.

Consistent with adjusted comparisons, absenteeism, presenteeism, and overall work productivity impairments did not differ significantly between those with or without N/V. Adjusted results revealed greater activity impairment experienced by migraineurs with $\mathrm{N} / \mathrm{V}$ relative to migraineurs without N/V (adjusted mean \% $=37.73$ [CI: $35.62-39.97]$ vs 35.12 [CI: 33.18-37.18], $p=0.002$ ).

\section{Health care resource use}

Unadjusted comparisons showed that those with N/V visited any HCP and the ER in the past 6 months more often than those without N/V. There were no significant differences in 
Table I Demographics and health characteristics among migraineurs with or without N/V

\begin{tabular}{|c|c|c|c|c|c|}
\hline \multicolumn{2}{|c|}{ Demographics and health characteristics } & \multicolumn{3}{|c|}{ Migraineurs } & \multirow[t]{2}{*}{ *p-value } \\
\hline & & $\begin{array}{l}\text { Total } \\
\mathrm{N}=7,855\end{array}$ & $\begin{array}{l}\text { With N/V } \\
(n=4,528)\end{array}$ & $\begin{array}{l}\text { Without N/V } \\
(n=3,327)\end{array}$ & \\
\hline \multicolumn{2}{|l|}{ Age, mean (SD), years } & $41.81(14.04)$ & $41.17(13.19)$ & $42.70(15.07)$ & $<0.001$ \\
\hline \multirow[t]{2}{*}{ Gender, n (\%) } & Female & $5,766(73.4)$ & $3,736(82.5)$ & $2,030(6 I .0)$ & $<0.001$ \\
\hline & Male & $2,089(26.6)$ & $792(17.5)$ & $1,297(39.0)$ & \\
\hline \multirow[t]{4}{*}{ Race/ethnicity, n (\%) } & Non-Hispanic white & 5,635 (7I.7) & $3,409(75.3)$ & $2,226(66.9)$ & $<0.001$ \\
\hline & Non-Hispanic black & $789(10.0)$ & $310(6.8)$ & $479(14.4)$ & \\
\hline & Hispanic & $811(10.3)$ & $438(9.7)$ & 373 (1 I.2) & \\
\hline & Other ethnicity & $620(7.9)$ & $37 \mid(8.2)$ & $249(7.5)$ & \\
\hline \multirow[t]{2}{*}{ Education, n (\%) } & Less than 4-year degree & $4,927(62.7)$ & $2,867(63.3)$ & $2,060(61.9)$ & 0.205 \\
\hline & 4-year degree or higher & $2,928(37.3)$ & $\mathrm{I}, 66 \mathrm{I}(36.7)$ & $1,267(38.1)$ & \\
\hline \multirow{5}{*}{$\begin{array}{l}\text { Annual household income, } \\
\text { n (\%) }\end{array}$} & $<$ US $\$ 25,000$ & I,734 (22.1) & $1,012(22.3)$ & $722(21.7)$ & 0.007 \\
\hline & US $\$ 25,000-U S \$ 50,000$ & $2,175(27.7)$ & I,3 I I (29.0) & $864(26.0)$ & \\
\hline & US $\$ 50,000-U S \$ 75,000$ & $1,563(19.9)$ & $893(19.7)$ & $670(20.1)$ & \\
\hline & US $\$ 75,000$ or more & $1,912(24.3)$ & $1,043(23.0)$ & $869(26.1)$ & \\
\hline & Declined to answer & $47 I(6.0)$ & $269(5.9)$ & $202(6.1)$ & \\
\hline \multirow[t]{5}{*}{ BMI category, n (\%) } & Underweight & $182(2.3)$ & $113(2.5)$ & $69(2.1)$ & 0.019 \\
\hline & Normal weight & $2,625(33.4)$ & I,492 (33.0) & I,I33 (34.I) & \\
\hline & Overweight & $2,118(27.0)$ & I, I 75 (25.9) & $943(28.3)$ & \\
\hline & Obese & $2,73 \mathrm{I}(34.8)$ & $1,624(35.9)$ & $1,107(33.3)$ & \\
\hline & Unknown & $199(2.5)$ & $124(2.7)$ & $75(2.3)$ & \\
\hline \multirow[t]{3}{*}{ Smoking status, n (\%) } & Current Smoker & $\mathrm{I}, 686(2 \mathrm{I} .5)$ & $985(21.8)$ & $70 \mathrm{I}(2 \mathrm{I} . \mathrm{I})$ & $0.55 I$ \\
\hline & Former Smoker & $2,093(26.6)$ & I,2I7 (26.9) & $876(26.3)$ & \\
\hline & Never Smoker & $4,076(51.9)$ & $2,326(51.4)$ & $1,750(52.6)$ & \\
\hline \multirow[t]{2}{*}{ Drink alcohol, n (\%) } & No & $2,578(32.8)$ & I,52I (33.6) & I,057 (31.8) & 0.090 \\
\hline & Yes & $5,277(67.2)$ & $3,007(66.4)$ & $2,270(68.2)$ & \\
\hline Exercise $20+$ minutes in past & 0 times & $2,525(32.1)$ & $\mathrm{I}, 495(33.0)$ & $1,030(31.0)$ & 0.054 \\
\hline month, $\mathrm{n}(\%)$ & I+ times & $5,330(67.9)$ & $3,033(67.0)$ & $2,297(69.0)$ & \\
\hline \multicolumn{2}{|c|}{ Number of migraines in the past 30 days, mean (SD) } & $3.27(5.33)$ & $3.31(5.23)$ & $3.22(5.47)$ & 0.482 \\
\hline \multicolumn{2}{|c|}{$\mathrm{CCl}$, mean $(\mathrm{SD})$} & $0.55(1.26)$ & $0.55(1.15)$ & $0.54(1.40)$ & 0.725 \\
\hline
\end{tabular}

Note: ${ }^{*} p$-value represents comparison of with $N / V$ vs without N/V. Values in bold represent significant pairwise differences at $p<0.05$ from tests of column proportions (z tests) for categorical variables.

Abbreviations: $\mathrm{N} / \mathrm{V}$, nausea/vomiting; $\mathrm{BMI}$, body mass index; $\mathrm{CCl}$, Charlson comorbidity index; SD, standard deviation.

the number of times hospitalization occurred between the migraine groups.

After adjusting for covariates, only the mean number of ER visits was significantly higher (by about 26\%) among migraineurs with $\mathrm{N} / \mathrm{V}$ than without $\mathrm{N} / \mathrm{V}$ (adjusted mean $=0.48$ [CI: $0.41-0.57$ ] vs 0.38 [CI: $0.32-0.45$ ], $p=0.001$ ) (Figure 3); the mean number of HCP visits and hospitalizations were comparable.

\section{Direct and indirect costs}

Unadjusted comparisons revealed only differences on annual ER visit costs, such that those with N/V incurred higher annual ER costs than those without N/V. Consistent with unadjusted results, after adjusting for covariates, migraineurs with $\mathrm{N} / \mathrm{V}$ compared with those without N/V incurred higher annual ER costs (adjusted mean $=$ US $\$ 1,499$ [CI: US\$1,249-US\$1,798] vs US\$1,187 [CI: US\$986US $\$ 1,427], p=0.002$ ) (Table 3). Annual costs associated with absenteeism (US\$2,290 [CI: US\$1,676-US\$3,130] vs US\$1,791 [CI: US\$1,299-US\$2,469], $p=0.046)$ and presenteeism (US\$7,954 [CI: US $\$ 7,063-\mathrm{US} \$ 8,956$ ] vs US $\$ 7,235$ [CI: US $\$ 6,442-U S \$ 8,126$ ], $p=0.040$ ) were also higher, resulting in higher annual indirect costs (US\$10,344 [CI: US\$9,146-US\$11,699] vs US\$9,218 [CI: US\$8,160US $\$ 10,412], p=0.016$ ) among those with $\mathrm{N} / \mathrm{V}$ relative to those without N/V (Table 3). Additionally, annual total costs were also higher among migraineurs with $\mathrm{N} / \mathrm{V}$ than those without N/V (US\$25,644 [CI: US\$23,491-US\$27,995] vs US $\$ 23,831$ [CI: US $\$ 21,819-U S \$ 26,029] p=0.037$ ) (Table 3).

\section{Discussion}

Migraine carries a substantial impact on day-to-day functioning and overall quality of life. ${ }^{14-16}$ The reported clinical characteristics of migraine, and the fact that its prevalence and incidence peak in middle life when an individual is likely to be more actively engaged in work, social, and family duties, explains why migraine can cause a substantial negative impact on patient lives. ${ }^{6}$ Most notably, our 
Table 2 Clinical characteristics and treatment history among migraineurs with or without N/V

\begin{tabular}{|c|c|c|c|c|}
\hline \multirow[t]{2}{*}{ Migraine characteristics/treatment } & \multicolumn{3}{|c|}{ Migraineurs } & \multirow[t]{2}{*}{ *p-value } \\
\hline & Total $\mathbf{N}=7,855$ & With N/V $(n=4,528)$ & Without N/V (n=3,327) & \\
\hline \multicolumn{5}{|l|}{ Migraine Symptoms, n (\%) } \\
\hline Aura & $1,940(24.7)$ & $1,346(29.7)$ & $594(17.9)$ & $<0.001$ \\
\hline Moderate to severe pain & $6,163(78.5)$ & $3,908(86.3)$ & $2,255(67.8)$ & $<0.001$ \\
\hline Nausea and/or vomiting & $4,528(57.6)$ & $4,528(100.0)$ & $0(0.0)$ & $<0.001$ \\
\hline Pulsating, throbbing, or pounding pain & $5,420(69.0)$ & $3,566(78.8)$ & $\mathrm{I}, 854(55.7)$ & $<0.001$ \\
\hline $\begin{array}{l}\text { Pain is worse on one side of your head } \\
\text { or occurs on one side of your head only }\end{array}$ & $4,146(52.8)$ & $2,732(60.3)$ & $\mathrm{I}, 4 \mathrm{I} 4(42.5)$ & $<0.001$ \\
\hline $\begin{array}{l}\text { Pain is made worse by routine activities } \\
\text { such as walking or climbing stairs }\end{array}$ & $3,167(40.3)$ & $2,330(51.5)$ & $837(25.2)$ & $<0.001$ \\
\hline $\begin{array}{l}\text { Bothered by or unusually sensitive } \\
\text { to light }\end{array}$ & $5,620(71.5)$ & $3,818(84.3)$ & $\mathrm{I}, 802(54.2)$ & $<0.001$ \\
\hline $\begin{array}{l}\text { Bothered by or unusually sensitive } \\
\text { to sound }\end{array}$ & $4,567(58.1)$ & $3,270(72.2)$ & $\mathrm{I}, 297(39.0)$ & $<0.001$ \\
\hline $\begin{array}{l}\text { See spots, flashing lights, or heat waves } \\
\text { before or during the migraine }\end{array}$ & $3,050(38.8)$ & $2,104(46.5)$ & $946(28.4)$ & $<0.001$ \\
\hline $\begin{array}{l}\text { Lasts for at least } 4 \text { hours but not more } \\
\text { than } 72 \text { hours if untreated }\end{array}$ & $4,146(52.8)$ & $2,902(64.1)$ & $\mathrm{I}, 244(37.4)$ & $<0.001$ \\
\hline \multicolumn{5}{|l|}{ Severity of migraines, $\mathrm{n}(\%)$} \\
\hline Mild & $1,255(15.6)$ & $404(8.9)$ & $82 I(24.7)$ & $<0.001$ \\
\hline Moderate & $3,974(50.6)$ & $2,131(47.1)$ & $\mathrm{I}, 843(55.4)$ & \\
\hline Severe & $2,656(33.8)$ & $1,993(44.0)$ & $663(19.9)$ & \\
\hline \multicolumn{5}{|l|}{ Preventative migraine treatments, $\mathrm{n}(\%)$} \\
\hline Botox (botulinum toxin type a ) & $83(1.1)$ & $47(1.0)$ & $36(1.1)$ & 0.850 \\
\hline Depakote (valproate semisodium) & $56(0.7)$ & $27(0.6)$ & $29(0.9)$ & 0.152 \\
\hline DepakoteER (divalproex sodium) & $35(0.4)$ & $16(0.4)$ & $19(0.6)$ & 0.152 \\
\hline Inderal (propranolol) & $102(1.3)$ & $72(1.6)$ & $30(0.9)$ & 0.008 \\
\hline Neurontin (gabapentin) & $|8|(2.3)$ & $102(2.3)$ & $79(2.4)$ & 0.722 \\
\hline Topamax (topiramate) & $512(6.5)$ & $329(7.3)$ & $183(5.5)$ & 0.002 \\
\hline \multicolumn{5}{|l|}{ Acute migraine treatments } \\
\hline Alsuma (sumatriptan succinate) & $55(0.7)$ & $20(0.4)$ & $35(I . I)$ & 0.001 \\
\hline Amerge (naratriptan) & $40(0.5)$ & $26(0.6)$ & $14(0.4)$ & 0.345 \\
\hline Axert (almotriptan) & $44(0.6)$ & $23(0.5)$ & $21(0.6)$ & 0.470 \\
\hline Cambia (diclofenac potassium) & $47(0.6)$ & $15(0.3)$ & $32(1.0)$ & $<0.001$ \\
\hline $\begin{array}{l}\text { Fioricet (butalbital/acetaminophen/ } \\
\text { caffeine) }\end{array}$ & $344(4.4)$ & $234(5.2)$ & $110(3.3)$ & $<0.001$ \\
\hline Fiorinal (aspirin/butalbital/caffeine) & $|4|(1.8)$ & $76(1.7)$ & $65(2.0)$ & 0.364 \\
\hline Frova (frovatriptan) & $63(0.8)$ & $35(0.8)$ & $28(0.8)$ & 0.736 \\
\hline ImitrexInjection (sumatriptan) & $130(1.7)$ & $106(2.3)$ & $24(0.7)$ & $<0.001$ \\
\hline ImitrexTablets (sumatriptan) & $729(9.3)$ & $507(11.2)$ & $222(6.7)$ & $<0.001$ \\
\hline ImitrexNasalSpray (sumatriptan) & $72(0.9)$ & $47(1.0)$ & $25(0.8)$ & 0.188 \\
\hline $\begin{array}{l}\text { Midrin (acetaminophen/isometheptene/ } \\
\text { dichloralphenazone) }\end{array}$ & $207(2.6)$ & $105(2.3)$ & $102(3.1)$ & 0.041 \\
\hline Maxalt (rizatriptan) & $378(4.8)$ & $247(5.5)$ & $|3|(3.9)$ & 0.002 \\
\hline Relpax (eletriptan) & $176(2.2)$ & $106(2.3)$ & $70(2.1)$ & 0.483 \\
\hline Sumatriptan (sumatriptan) & $233(3.0)$ & $163(3.6)$ & $70(2.1)$ & $<0.001$ \\
\hline $\begin{array}{l}\text { Treximet (sumatriptan/naproxen } \\
\text { sodium) }\end{array}$ & $81(1.0)$ & $45(1.0)$ & $36(1.1)$ & 0.702 \\
\hline ZomigTablets (zolmitriptan) & $159(2.0)$ & $98(2.2)$ & $61(1.8)$ & 0.304 \\
\hline ZomigNasalSpray (zolmitriptan) & $24(0.3)$ & $15(0.3)$ & $9(0.3)$ & 0.630 \\
\hline Other & $761(9.7)$ & $432(9.5)$ & $329(9.9)$ & 0.606 \\
\hline
\end{tabular}

Note: *p-value represents comparison of with N/V vs without N/V. Values in bold represent significant pairwise differences at $p<0.05$ from tests of column proportions (z tests) for categorical variables.

Abbreviations: N/V, nausea/vomiting; BMI, body mass index; $\mathrm{CCl}$, Charlson comorbidity index. 


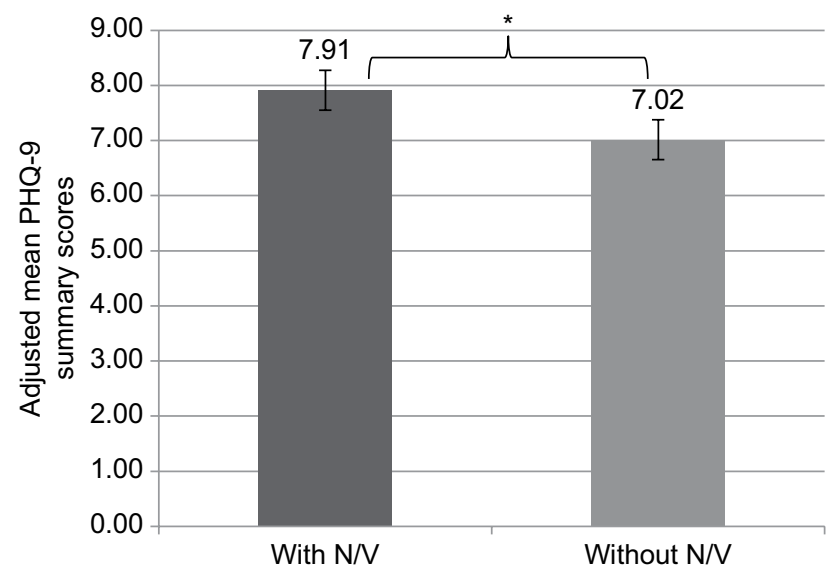

Figure I Mean PHQ-9 summary scores among migraineurs with or without N/V, adjusting for covariates.

Notes: Error bars depict $95 \%$ confidence intervals. Covariates included: age, gender, ethnicity, education, income, $\mathrm{BMI}, \mathrm{CCl}$, exercise, smoking, and alcohol use. ${ }^{*} p<0.05$ represents comparison of with N/V vs without N/V.

Abbreviations: PHQ-9, 9-item Patient Health Questionnaire; N/V, nausea/ vomiting; $\mathrm{BMI}$, body mass index; $\mathrm{CCl}$, Charlson Comorbidity Index.

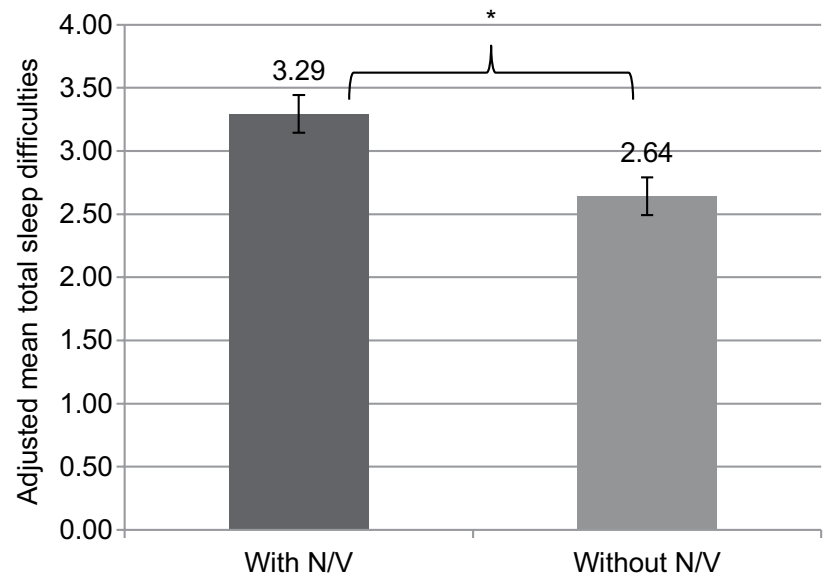

Figure 2 Mean total sleep difficulties among migraineurs with or without N/V adjusting for covariates.

Notes: Error bars depict $95 \%$ confidence intervals. Covariates included: age, gender, ethnicity, education, income, $\mathrm{BMI}, \mathrm{CCl}$, exercise, smoking, and alcohol use ${ }^{*} p<0.05$ represents comparison of with $N / V$ vs without N/V.

Abbreviations: $\mathrm{N} / \mathrm{V}$, nausea/vomiting; $\mathrm{BMI}$, body mass index; $\mathrm{CCl}$, Charlson Comorbidity Index.

findings revealed that migraineurs with $\mathrm{N} / \mathrm{V}$ reported greater burden on sleep, depression, and activity impairment than those without N/V. Additionally, migraineurs with N/V had $26 \%$ more ER visits and incurred higher indirect and total costs compared with migraineurs without N/V. Thus, the current study provides additional important insight into the humanistic and economic impact of migraines and the burden imposed by nausea and vomiting. Little research exists detailing the health and economic consequences of this additional symptom burden.

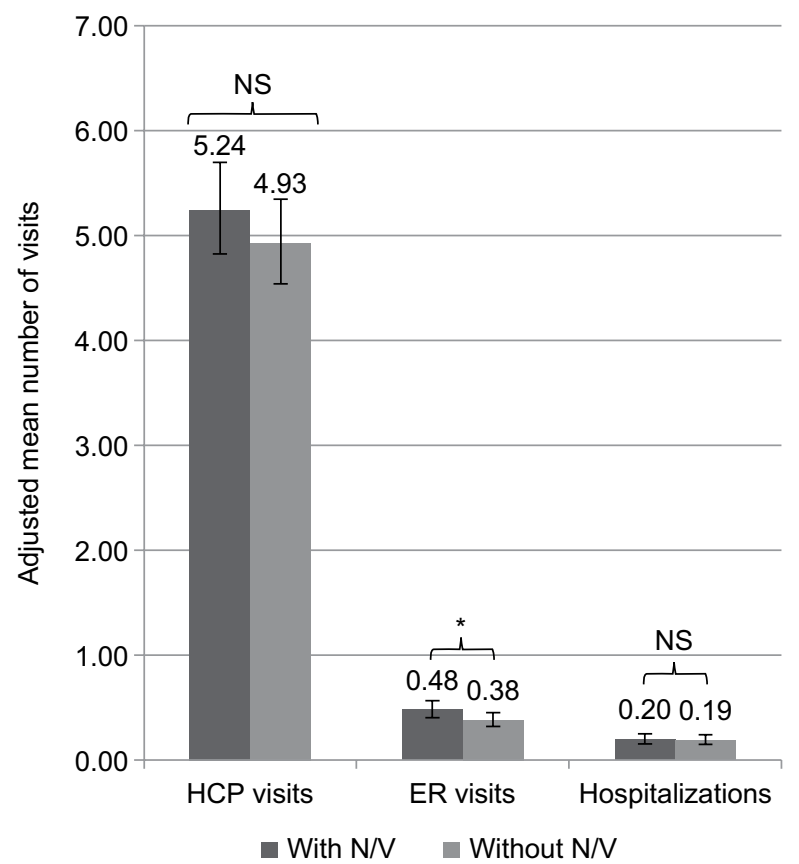

Figure 3 Mean HRU visits among migraineurs with or without $N / V$, adjusting for covariates.

Notes: Error bars depict $95 \%$ confidence intervals. Covariates included: age, gender, ethnicity, education, income, $\mathrm{BMI}, \mathrm{CCl}$, exercise, smoking, and alcohol use. ${ }^{*} p<0.05$ represents comparison of with $N / V$ vs without $N / V$.

Abbreviations: HCP, health care provider; ER, emergency room; HRU, health care resource use; $\mathrm{N} / \mathrm{V}$, nausea/vomiting; $\mathrm{BMI}$, body mass index; $\mathrm{CCl}$, Charlson Comorbidity Index; NS, not significant.

In the current study, 58\% reported experiencing N/V, slightly fewer than in previous studies that cited rates of $73 \%-90 \%$ for nausea and $29 \%-70 \%$ for vomiting among migraineurs. ${ }^{4-6}$ In a survey of nearly 30,000 individuals, Lipton et $\mathrm{al}^{6}$ found that among those with migraine headaches, $73 \%$ reported experiencing nausea and $29 \%$ reported vomiting. The demographic characteristics of migraineurs were similar to the current study in regard to race/ethnicity (majority non-Hispanic white) and income distribution; however, the current study did possess a greater percentage of female respondents. Importantly, the way in which migraine was defined differed between the Lipton et $\mathrm{l}^{6}{ }^{6}$ study and the present study. Lipton et $\mathrm{al}^{6}$ used the 1998 IHS criteria for migraine with or without aura, whereas the current study defined migraine from self-reported physician diagnosis. This likely explains the differences in rates on migraine with $\mathrm{N} / \mathrm{V}$ between the two studies.

Despite the paucity of previous research in this domain, the primary findings of this study were consistent with previous work and emphasize the burden and consequences of N/V in the context of migraine. In a further large-scale study of migraine sufferers, Lipton et $\mathrm{al}^{20}$ reported increases in health care costs among those with N/V compared with 
Table 3 Costs comparisons among migraineurs with N/V vs without $N / V$, adjusting for covariates

\begin{tabular}{|c|c|c|c|c|c|c|c|c|c|}
\hline \multirow[t]{3}{*}{ Costs } & \multicolumn{8}{|c|}{ Migraineurs } & \multirow[t]{3}{*}{$* p$-value } \\
\hline & \multicolumn{4}{|c|}{ With N/V $(n=4,528)$} & \multicolumn{4}{|c|}{ Without N/V $(n=3,327)$} & \\
\hline & Mean & SE & LCL & UCL & Mean & SE & LCL & UCL & \\
\hline \multicolumn{10}{|l|}{ Direct costs } \\
\hline HCP costs & 10,784 & 459 & 9,921 & $\mathrm{II}, 722$ & 10,185 & 431 & 9,375 & 11,065 & 0.083 \\
\hline ER costs & $\mathrm{I}, 499$ & 139 & 1,249 & $\mathrm{I}, 798$ & $\mathrm{I}, 187$ & 112 & 986 & $\mathrm{I}, 427$ & 0.002 \\
\hline Hospitalization costs & 6,132 & 801 & 4,747 & 7,921 & 5,728 & 777 & 4,390 & 7,473 & 0.512 \\
\hline Total & 19,662 & $|, 02|$ & 17,759 & 21,769 & 18,398 & 966 & 16,598 & 20,393 & 0.103 \\
\hline \multicolumn{10}{|l|}{ Indirect costs } \\
\hline Absenteeism & 2,290 & 365 & 1,676 & 3,130 & I,79| & 293 & 1,299 & 2,469 & 0.046 \\
\hline Presenteeism & 7,954 & 482 & 7,063 & 8,956 & 7,235 & 429 & 6,442 & 8,126 & 0.040 \\
\hline Total & 10,344 & 650 & 9,146 & 11,699 & 9,218 & 573 & 8,160 & $10,4 \mid 2$ & 0.016 \\
\hline Total costs & 25,644 & $\mathrm{I}, \mathrm{I} 47$ & 23,491 & 27,995 & 23,831 & 1,073 & 21,819 & 26,029 & 0.037 \\
\hline
\end{tabular}

Note: Covariates included: age, gender, ethnicity, education, income, BMI, CCl, exercise, smoking, and alcohol use. ${ }^{*} p$-value represents comparison of with $\mathrm{N} / \mathrm{V}$ vs without N/V.

Abbreviations: N/V, nausea/vomiting; HCP, health care provider; ER, emergency room; SE, standard error; LCL, lower confidence interval; UCL, upper confidence interval; $\mathrm{BMI}$, body mass index; $\mathrm{CCl}$, Charlson Comorbidity Index.

those without. As in the current study, migraine sufferers with nausea reported higher rates of ER visits, as well as increased costs of care, notably a threefold increase in outpatient service care. Further, the current findings were consistent with previous reports of increased rates of depression among migraineurs compared with the general population. ${ }^{12}$ However, the current study adds to this literature by showing that migraineurs with $\mathrm{N} / \mathrm{V}$ have higher rates of depression compared with those without $\mathrm{N} / \mathrm{V}$, thus providing insight into the additional burden specific to N/V.

The current study identifies an important area of opportunity to focus treatment efforts and should raise awareness among providers who manage the care of migraineurs. Future research may focus on examining the specific migraine treatments among migraineurs with N/V in order to better understand the specific unmet needs, such as patients' ratings of importance of treatment attributes, their satisfaction and expectations of a treatment, and the impact of their condition as well as satisfaction with treatment on adherence and associated health outcomes. Providers may be unaware of the substantial impact of symptoms such as N/V on quality of life and health outcomes, and thus associated symptoms may not receive the full attention of medical providers. Additionally, patients who are seeking assistance for their migraine may be unwilling to report symptoms such as N/V so as to not detract from focus on the treatment of migraine, or may perceive such symptoms to be unavoidable.

The successful management of N/V may have a measurable and important impact on the quality of life of sufferers, as well as potentially reducing the economic burden of migraines in society as a whole. Indeed, given this and previous studies noted earlier, findings suggest that N/V can be associated with an increased rate of health care utilization, ${ }^{20}$ and thus the effective management of symptomatology would likely lead to better patient outcomes.

\section{Strengths and limitations}

The current study includes a number of important strengths, including sampling data from a real-world population of migraine sufferers and relying upon patient-reported outcomes to assess burden. In addition, this study fills an important gap in the literature regarding the specific health and economic burden associated with $\mathrm{N} / \mathrm{V}$ in the context of migraines.

It is also important to recognize the limitations of the current study in interpreting results. Data on migraine diagnosis and other variables were self-reported and could not be verified by respondents' medical charts. The study's measure of depression (PHQ-9) is a screener for depression, and thus does not represent diagnosed depression. Similarly, sleep difficulties represent sleep problems experienced by the respondents and not diagnoses of specific sleep conditions. Thus, these measures may capture less severe depression or sleep difficulties than would diagnoses. Nevertheless, there may also be patients who fail to report such symptoms to their physicians but are yet identified as having these issues based on their responses on these questionnaires. The study also relied upon respondents recalling their experiences relating to migraine, and thus there may be a degree of recall bias. Additionally, while patient-reported data can provide meaningful information about patients' experience with their disease, they are subjective and how patients interpret their condition may not completely align with clinical definitions. 
For example, the clinical definition of a migraine is that the migraine lasts for at least 4 hours but not more than 72 hours if untreated, and yet only $52.8 \%$ of the total sample reported having this symptom. This response may be a reflection of some patients taking into account that they are currently treated $(40.9 \%$ of the sample are treated) and therefore do not feel they experience this symptom even though they are diagnosed with migraines. While efforts were made to establish a representative data set and assess all relevant variables, there may be bias present, and thus caution should be used in extrapolating from this study. Although a number of respondent characteristics were adjusted for, there may be additional variables that were not adjusted for that may have biased the results. For example, respondents' comorbid conditions not included in the analysis may have an impact on the outcomes examined, such as ER visits and associated costs. However, in multivariable analyses we controlled for the most impactful comorbidities using the CCI. A potential confounding factor for the association of depression and $\mathrm{N} / \mathrm{V}$ is that selective serotonin-reuptake inhibitor (SSRI) antidepressants can cause N/V in depressed patients. Further research is needed to tease apart the relationship between $\mathrm{N} / \mathrm{V}$ and depression among migraineurs. As total HRU and work productivity costs were extrapolated from the MEPS and BLS, which were based on general population data, cost estimates are likely biased. Additionally, total costs (direct and indirect costs combined) are a conservative estimate of costs since unemployed respondents do not have indirect costs included in the calculation. Finally, the NHWS was designed to be representative of the general US adult population (ie, age, gender, and ethnicity); however, representativeness of the migraine population may be skewed. For example, it may be possible that NHWS favors participation from patients with less severe disease as those with very severe conditions would not have the energy or cognitive capacity to participate in an online survey. Such an explanation may be the reason why the percentage of self-reported N/V in the current study is lower compared with other population-based migraine studies.

\section{Conclusion}

Migraines with N/V are associated with greater burden across measures of sleep, depressive symptoms, and activity impairment compared with migraines without N/V. Additionally, migraines with N/V are associated with greater economic burden as demonstrated by higher indirect and total costs. These findings emphasize the importance of addressing N/V in the context of migraine. Further research is needed to demonstrate whether successful symptom management of
$\mathrm{N} / \mathrm{V}$ in migraine may help improve patient quality of life and reduce the economic burden of migraine on society.

\section{Acknowledgments}

The authors acknowledge the literature review and editing assistance of Errol J Philip, PhD, on behalf of Kantar Health, with funding from Teva Pharmaceuticals. Portions of this research were presented as a Poster (PF52: "Humanistic and Economic Burden of Nausea and/or Vomiting among Migraine Sufferers: Results from the National Health and Wellness Survey") at the 57th Annual Scientific Meeting of the American Headache Society, and published in "Headache: The Journal of Head and Face Pain," June, 2015. http:// onlinelibrary.wiley.com/doi/10.1111/head.12600/abstract.

\section{Author contributions}

Kavita Gajria and Sanjay K Gandhi conceptualized the rationale and design of the study. Lulu K Lee and Natalia M Flores participated in statistical analyses and interpretation of study results. All authors participated in interpretation of the study results, revisions of earlier manuscript drafts, and approved the final manuscript.

\section{Disclosure}

Kavita Gajria, Ernesto Aycardi, and Sanjay Gandhi are fulltime employees of Teva Pharmaceuticals, which provided funding for the study. Lulu $\mathrm{K}$ Lee is a full-time employee of Kantar Health, which received funding from Teva Pharmaceuticals to conduct this study. Natalia M Flores was an employee of Kantar Health when this study was conducted. The authors report no other conflicts of interest in this work.

\section{References}

1. Noseda R, Burstein R. Migraine pathophysiology: anatomy of the trigeminovascular pathway and associated neurological symptoms, cortical spreading depression, sensitization, and modulation of pain. Pain. 2013;154(Suppl 1):S44-S53.

2. American Migraine Foundation. Types of Migraines. [cited 2016 February]. Available from: https://americanmigrainefoundation. org/living-with-migraines/types-of-headachemigraine/. Accessed February 14, 2017.

3. American Headache Society. Patient Education. [cited 2016]. Available from: https://americanheadachesociety.org/patient-education/.

4. Silberstein SD. Migraine symptoms: results of a survey of self-reported migraineurs. Headache. 1995;35:387-396.

5. Mateos V, Pareja JA, Pacual J. Tratado de Cefaleas. [Headache Treatment]. 5th ed. Madrid, Spain: Luzán; 2009.Spanish.

6. Lipton RB, Stewart WF, Diamond S, Diamond ML, Reed M. Prevalence and burden of migraine in the United States: data from the American Migraine Study II. Headache. 2001;41:646-657.

7. Buse DC, Loder EW, Gorman JA, et al. Sex differences in the prevalence, symptoms, and associated features of migraine, probable migraine and other severe headache: results of the American Migraine Prevalence and Prevention (AMPP) Study. Headache. 2013;53:1278-1299. 
8. Wang SJ, Chen PK, Fuh JL. Comorbidities of migraine. Front Neurol. 2010;1:16.

9. Scher AI, Bigal ME, Lipton RB. Comorbidity of migraine. Curr Opin Neurol. 2005;18:305-310.

10. Bhatia MS, Gupta R. Migraine: clinical pattern and psychiatric comorbidity. Ind Psychiatry J. 2012;21:18-21.

11. Malone CD, Bhowmick A, Wachholtz AB. Migraine: treatments, comorbidities, and quality of life, in the USA. J Pain Res. 2015;8:537-547.

12. Antonaci F, Nappi G, Galli F, Manzoni GC, Calabresi P, Costa A. Migraine and psychiatric comorbidity: a review of clinical findings. $J$ Headache Pain. 2011;12:115-125.

13. Seidel S, Hartl T, Weber M, et al; PAMINA Study Group. Quality of sleep, fatigue and daytime sleepiness in migraine - a controlled study. Cephalalgia. 2009;29(6):662-669.

14. Abu Bakar N, Tanprawate S, Lambru G, Torkamani M, Jahanshahi M, Matharu M. Quality of life in primary headache disorders: a review. Cephalalgia. 2016;36(1):67-91.

15. Brna P, Gordon K, Dooley J. Health-related quality of life among Canadians with migraine. J Headache Pain. 2007;8:43-48.

16. Terwindt GM, Ferrari MD, Tijhuis M, Groenen SM, Picavet HS, Launer LJ. The impact of migraine on quality of life in the general population: the GEM study. Neurology. 2000;55:624-629.

17. Raggi A, Leonardi M, Bussone G, D'Amico D. A 3-month analysis of disability, quality of life, and disease course in patients with migraine. Headache. 2013;53:297-309.

18. Stefane T, Napoleao AA, Sousa FA, Hortense P. Influência de tratamentos para enxaqueca na qualidade de vida: revisão integrativa de literatura. [Influence of treatments for migraine on quality of life: literature integrative review]. Rev Bras Enferm. 2012;65:353-360. Portuguese.

19. Burch RC, Loder S, Loder E, Smitherman TA. The prevalence and burden of migraine and severe headache in the United States: updated statistics from government health surveillance studies. Headache. 2015;55:21-34.
20. Lipton RB, Buse DC, Saiers J, Serrano D, Reed ML. Healthcare resource utilization and direct costs associated with frequent nausea in episodic migraine: results from the American Migraine Prevalence and Prevention (AMPP) Study. J Med Econ. 2013;16:490-499.

21. Lipton RB, Buse DC, Saiers J, Fanning KM, Serrano D, Reed ML. Frequency and burden of headache-related nausea: results from the American Migraine Prevalence and Prevention (AMPP) study. Headache. 2013;53:93-103.

22. Reed ML, Fanning KM, Serrano D, Buse DC, Lipton RB. Persistent frequent nausea is associated with progression to chronic migraine: AMPP study results. Headache. 2015;55:76-87.

23. Charlson ME, Pompei P, Ales KL, MacKenzie CR. A new method of classifying prognostic comorbidity in longitudinal studies: development and validation. J Chronic Dis. 1987;40:373-383.

24. Quan H, Li B, Couris CM, et al. Updating and validating the Charlson comorbidity index and score for risk adjustment in hospital discharge abstracts using data from 6 countries. Am J Epidemiol. 2011; 173:676-682.

25. Kroenke K, Spitzer RL. The PHQ-9: a new depression diagnostic and severity measure. Psychiatr Ann. 2002;32:509-521.

26. Kroenke K, Spitzer RL, Williams JB. The PHQ-9: validity of a brief depression severity measure. J Gen Intern Med. 2001;16: 606-613.

27. Reilly MC, Zbrozek AS, Dukes EM. The validity and reproducibility of a work productivity and activity impairment instrument. Pharmacoeconomics. 1993;4:353-365.

28. Agency for Healthcare Research and Quality. Medical Expenditure Panel Survey: Center for Financing, Access and Cost Trends; 2012. Available from: http://meps.ahrq.gov/mepsweb/index.jsp.

29. U.S. Bureau of Labor Statistics. Highlights of Women's Earnings in 2012. Available from: https://www.bls.gov/opub/reports/womens-earnings/ archive/womensearnings_2012.pdf.
Journal of Pain Research

\section{Publish your work in this journal}

The Journal of Pain Research is an international, peer reviewed, open access, online journal that welcomes laboratory and clinical findings in the fields of pain research and the prevention and management of pain. Original research, reviews, symposium reports, hypothesis formation and commentaries are all considered for publication.

\section{Dovepress}

The manuscript management system is completely online and includes a very quick and fair peer-review system, which is all easy to use. Visit http://www.dovepress.com/testimonials.php to read real quotes from published authors. 